\title{
XCL1 expression correlates with CD8-positive T cells infiltration and PD-L1 expression in squamous cell carcinoma arising from mature cystic teratoma of the ovary
}

\author{
Ryo Tamura ${ }^{1} \cdot{\text { Kosuke Yoshihara }(1)^{1} \cdot \text { Hirofumi Nakaoka }}^{2} \cdot$ Nozomi Yachida $^{1} \cdot$ Manako Yamaguchi $^{1} \cdot$ Kazuaki Suda $^{1}$. \\ Tatsuya Ishiguro $\mathbb{1}^{1} \cdot \mathrm{Koji}^{\mathrm{Nishino}}{ }^{1} \cdot$ Hiroshi Ichikawa ${ }^{3} \cdot \mathrm{Keiichi} \mathrm{Homma}^{4} \cdot$ Akira Kikuchi $^{5} \cdot$ Yutaka Ueda $^{6}$.

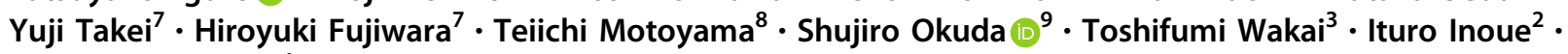 \\ Takayuki Enomoto ${ }^{1}$
}

Received: 7 November 2019 / Revised: 15 February 2020 / Accepted: 19 February 2020 / Published online: 2 March 2020

(c) The Author(s) 2020. This article is published with open access

\begin{abstract}
Molecular characteristics of carcinoma arising from mature cystic teratoma of the ovary (MCT) remain unclear due to its rarity. We analyzed RNA-sequencing data of 2322 pan-cancer [1378 squamous cell carcinomas (SCC), 6 adenosquamous carcinomas (ASC), and 938 adenocarcinomas (AC)] including six carcinomas arising from MCT (four SCCs, one ASC, and one AC). Hierarchical clustering and principal component analysis showed that gene expression profiles of carcinomas arising from MCT were different between each histological type and that gene expression profiles of SCCs arising MCT (MCT-SCCs) was apparently similar to those of lung SCCs. By epidermis-associated pathways activity based on gene set enrichment analysis, 1030 SCCs were divided into two groups: epidermis-signature high (head and neck, esophagus, and skin) and low (cervix, lung, and MCT). In addition to pan-SCC transcriptome analysis, cytokeratin profiling based on immunohistochemistry in the independent samples of 21 MCT-SCCs clarified that MCT-SCC dominantly expressed CK18, suggesting the origin of MCT-SCC was columnar epithelium. Subsequently, we investigated differentially expressed genes in MCT-SCCs compared with different SCCs and identified XCL1 was specifically overexpressed in MCT-SCCs. Through immunohistochemistry analysis, we identified XCL1 expression on tumor cells in 13/24 (54\%) of MCT-SCCs but not in MCTs. XCL1 expression was also significantly associated with the number of tumor-infiltrating CD8-positive T cells and PD-L1 expression on tumor cells. XCL1 produced by tumor cells may induce PD1/PD-L1 interaction and dysfunction of CD8-positive T cells in tumor microenvironment. XCL1 expression may be a novel biomarker for malignant transformation of MCT into SCC and a biomarker candidate for therapeutic response to an anti-PD1/PD-L1 therapy.
\end{abstract}

Supplementary information The online version of this article (https:// doi.org/10.1038/s41388-020-1237-0) contains supplementary material, which is available to authorized users.

Kosuke Yoshihara

yoshikou@med.niigata-u.ac.jp

1 Department of Obstetrics and Gynecology, Niigata University Graduate School of Medical and Dental Sciences, Niigata 9518510, Japan

2 Human Genetics Laboratory, National Institute of Genetics, Mishima 411-8540, Japan

3 Division of Digestive and General Surgery, Niigata University Graduate School of Medical and Dental Sciences, Niigata 9518510, Japan

4 Department of Pathology, Niigata Cancer Center Hospital, Niigata 951-8133, Japan
5 Department of Gynecology, Niigata Cancer Center Hospital, Niigata 951-8133, Japan

6 Department of Obstetrics and Gynecology, Osaka University School of Medicine, Suita 565-0871, Japan

7 Department of Obstetrics and Gynecology, Jichi Medical University, Shimotsuke 329-0498, Japan

8 Department of Molecular and Diagnostic Pathology, Niigata University Graduate School of Medical and Dental Sciences, Niigata 951-8510, Japan

9 Division of Bioinformatics, Niigata University Graduate School of Medical and Dental Sciences, Niigata 951-8510, Japan 


\section{Introduction}

Malignant transformation occurs in $~ 1-2 \%$ of mature cystic teratomas of the ovary (MCT), and the prognosis is poor at advanced stages [1]. Because of its rarity, there is no standard therapy for this disease. Malignant transformation of MCT has also been characterized by difficulty of preoperative diagnosis and histological diversity [1-3]. Squamous cell carcinoma (SCC) is the most common histological type in malignant transformation of MCT [1, 3], which is reasonable because skin epithelium is usually found in MCT [4]. Recently, Cooke et al. analyzed genomic alterations of 25 SCCs arising from MCT (MCT-SCC) by using a gene panel with 151 cancer-related genes and reported frequent alterations of TP53, PIK3CA, and $C D K N 2 A$ that are also frequently detected in other types of SCC [5]. However, the number of targeted genes in their study was limited, and other molecular characteristics of MCT-SCC such as transcriptome, proteome, and methylation profiles remain unclear. Specific biomarkers for the early diagnosis of malignant transformation and novel therapeutic targets, which are required in clinical practice, have not yet been identified.

The Cancer Genome Atlas (TCGA) Research Network has recently performed an extensive molecular characterization of SCC derived from four different anatomical sites (lung [6], head and neck [7], cervix [8], and esophagus [9]). Moreover, TCGA studies demonstrated molecular and biological similarities across SCCs derived from different organs $[10,11]$. In line with these molecular backgrounds, the clinical efficacy of anti-PD1 antibodies has been confirmed in SCC derived from lung [12], head and neck [13], cervix [14], esophagus [15], and skin tissues [16]. However, there were different immune subtypes in pan-SCCs, and the survival benefit of anti-PD1 antibodies was limited to a subset of SCC patients [17].

In this study, we investigated the molecular characteristics of carcinomas arising from MCT by performing comprehensive analyses of genomic and transcriptomic data and immunohistochemistry-based protein expression profiles. In particular, we compared gene expression profiles of MCT-SCCs with those of SCCs derived from other anatomical sites, leading to the development of novel therapeutic strategies for MCT-SCCs.

\section{Results}

\section{Patients and samples}

We prepared 27 carcinoma samples arising from MCT [23 SCCs, 3 adenosquamous carcinomas (ASC), and 1 adenocarcinoma (AC)]. The clinicopathological characteristics of the 27 carcinomas arising from MCT are shown in Table 1. The median onset age of all patients was 58 (33-79) years old, and 12/27 (44\%) patients were diagnosed at advanced stage (stage II-IV). Consistent with previous studies [1, 18], patients at an advanced stage (stage II-IV) had a poor prognosis (Supplementary Fig. 1). Because all cases include skin epithelial tissues, we evaluated histological findings of normal epithelium close to the cancer site and divided them into three types: cancer site close to skin epithelium (7/27, $26 \%$ ), cancer site close to not otherwise specified epithelium $(7 / 27,26 \%)$, and not available $(13 / 27,48 \%)$ (Supplementary Fig. 2). We could not find a sequential change from normal epithelium to carcinoma in situ in hematoxylin and eosin (H\&E) slides of our samples.

\section{Genomic alterations of SCC arising from MCT}

We used fresh frozen samples of four MCT-SCCs and one MCT-ASC to perform whole-exome and RNA sequencing. Exome sequencing data analysis showed that TP53 and PIK3CA were mutated in four out of five samples $(80 \%)$ (Supplementary Table 1), and other gene mutations were observed in less than half of MCT-SCC samples. When we determined pathogenic mutations based on mutations annotated in OncoKB [19] as pathogenic (oncogenic, likely oncogenic and predicted oncogenic) or COSMIC [20] as pathogenic (FATHHMM score $\geq 0.7$ ), 35 gene mutations were detected as "pathogenic" (Supplementary Table 2). Mutant allele expression was confirmed in 32 out of 35 pathogenic mutations by using RNA-sequencing data. Nine amplifications and two homozygous deletions were also detected, and $40 \%$ of samples harbored at least one copy number alteration (Supplementary Table 3). Moreover, we identified three in-frame fusion transcripts by using both PRADA [21] and FusionCatcher [22] (Supplementary Table 4). In particular, we found therapeutically targetable FGFR3-TACC3 fusion in one sample.

To increase the number of samples, we tried to sequence six formalin-fixed paraffin-embedded (FFPE) MCT-SCC samples using a gene panel containing 435 cancerassociated genes (CANCERPLEX ${ }^{\circledR}$ ) [23]. After a quality control check, we analyzed target-gene panel data in three samples. The details of oncogenic alterations identified by the gene panel are shown in Supplementary Table 5. We used a heatmap to illustrate the summary of pathogenic alterations in a total of eight samples (Fig. 1). TP53, PIK3CA, SETD2, and RB1 were frequently mutated in carcinomas arising from MCT $(87.5,50.0,25.0$, and $25.0 \%$ ), and at least one well-known oncogenic alteration was identified in all samples. Overall, genes associated with the PI3K-mTOR pathway, cell cycle pathway, protein kinase, and epigenetic regulator were frequently altered, and at least one druggable oncogenic alteration was detected in 


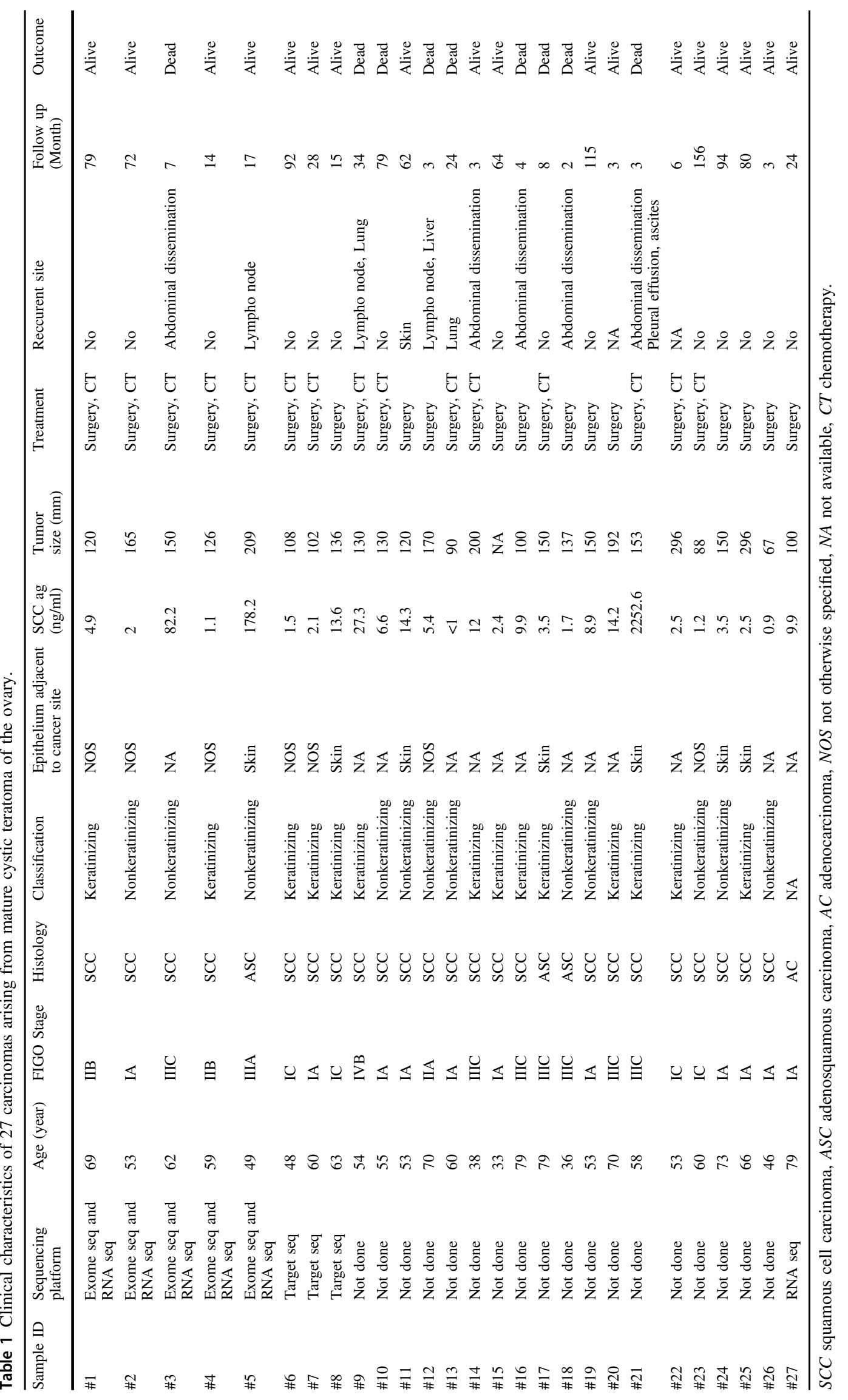




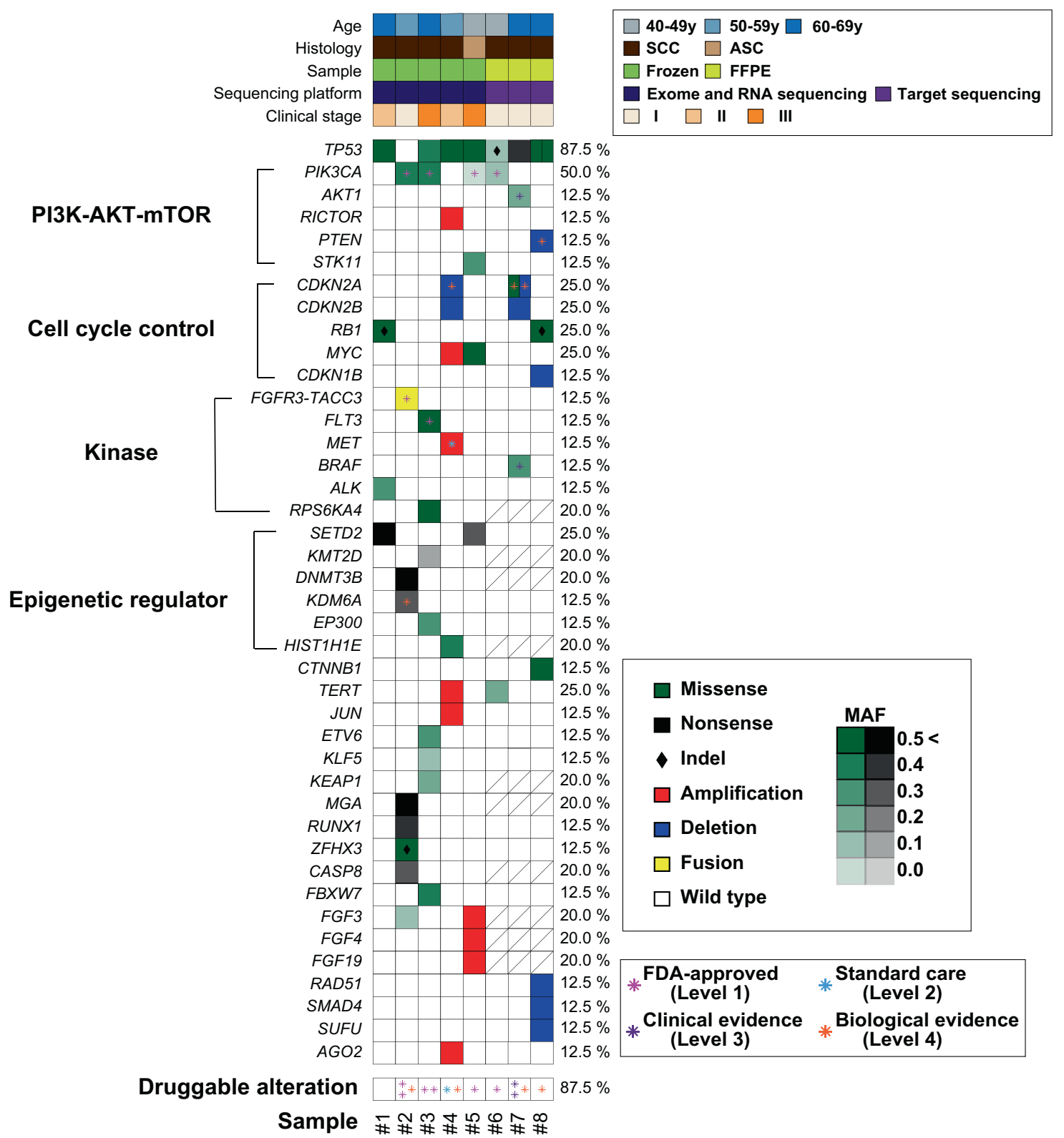

Fig. 1 Summary of pathogenetic alterations in MCT-SCCs. Heatmaps demonstrate both sample information (upper) and distribution of genetic alterations (lower). In the lower heatmap, color and density indicate the type and MAF of each somatic mutation, respectively.

seven samples (87.5\%) (Supplementary Table 6). We also evaluated tumor mutation burden (TMB) that was a promising biomarker for an immune checkpoint therapy $[24,25]$. The median TMB of eight samples was 4.2 mutations per megabase (range 0.8-9.9) (Supplementary Table 7).

Next, we compared the frequency of recurrent mutated genes (TP53, PIK3CA, SETD2, and RBI) in MCT-SCCs with that in publicly available pan-cancer data (Supplementary Fig. 3). TP53 and PIK3CA were also commonly mutated in SCC from other anatomical sites. However, the
Black diamonds represent indels. Asterisks indicate druggable alterations annotated in OncoKB on August 6, 2019. An oblique line in a square indicates that data are not available.

frequencies of PIK3CA (E542K and E545K mutations), the $S E T D 2$ nonsense mutation, and the $R B 1$ frameshift mutation were relatively high in MCT-SCC compared with SCC or $\mathrm{AC}$ arising in other anatomical sites.

\section{Gene expression profiling of SCC arising from MCT}

We used RNA-sequencing data to compare gene expression profiles among six carcinomas arising from MCT (four SCCs, one ASC, and one AC), nine cutaneous SCCs, and 2307 TCGA samples (1365 SCCs, 5 ASCs, and 937 ACs). 
a

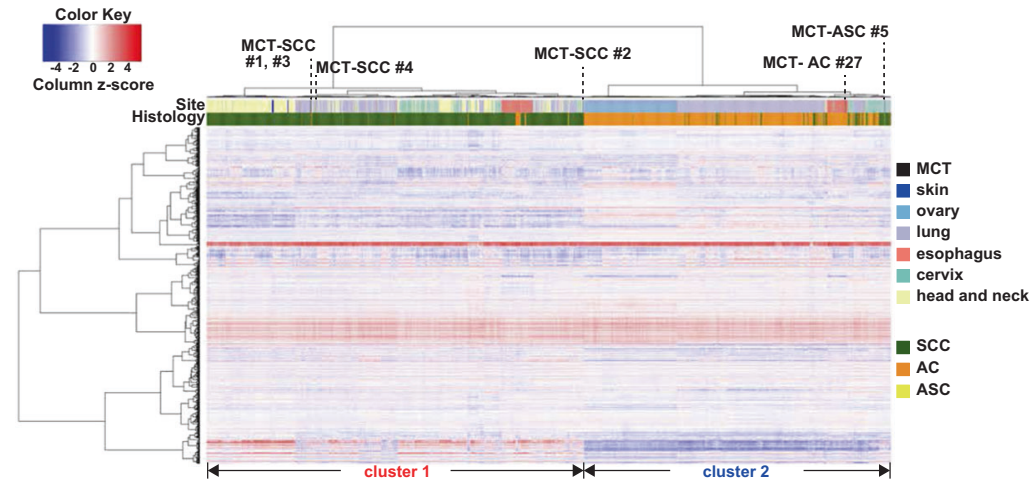

C
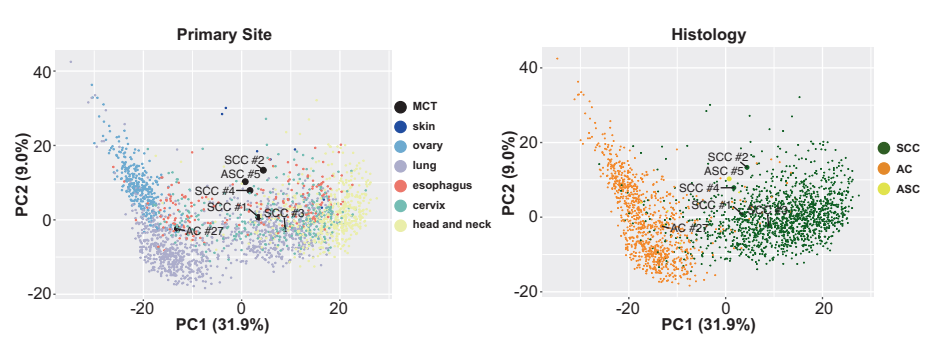

b

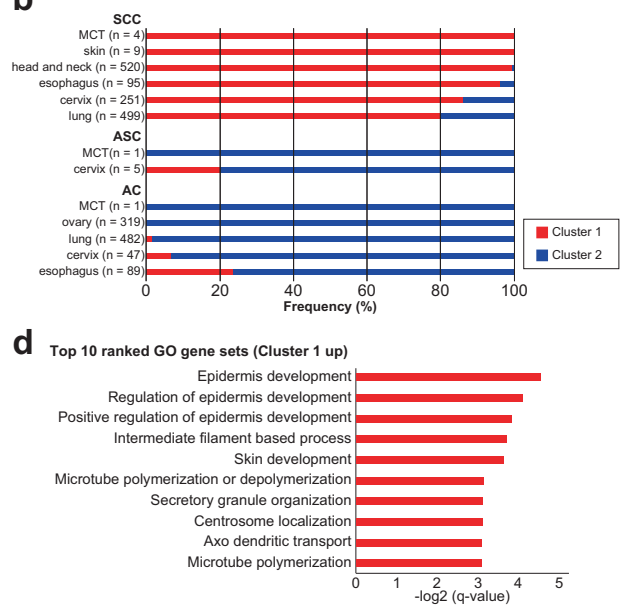

Top 10 ranked HALLMARK gene sets (Cluster 1 up)

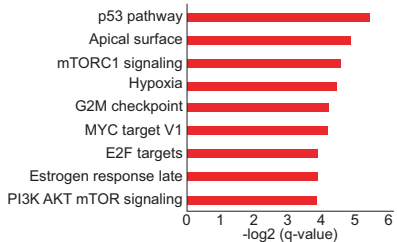

Fig. 2 Gene expression profile of carcinoma arising from MCT compared with pan-cancer. a A heatmap demonstrates the hierarchical clustering using the top 1000 variable genes of all samples $(n=2322)$. The color bar and legend show the primary site and histology of the samples, respectively. b The distribution of clusters 1 and 2 per tumor type stratified by histological type is shown. $\mathbf{c}$ PCA using

We extracted the top 1000 variable genes of all samples $(n=2322)$ to perform hierarchical clustering analysis (Fig. 2a). As a result, samples were divided into two groups that corresponded to histological types (SCC or AC). All MCTSCCs were classified in SCC-dominant cluster 1, and one MCT-AC was classified in AC-dominant cluster 2, which is consistent with histological types of carcinoma arising from MCT. The distribution of clusters 1 and 2 per tumor type stratified by histological type is shown in Fig. 2b. Intriguingly, MCT-ASC was classified into a subcluster of cluster 2 composed of both SCC and AC samples, and four out of five cervical ASCs were also classified in the same subcluster. Principal component analysis (PCA) using the same variable 1000 genes demonstrated a clear distinction between SCC and AC (Fig. 2c). Then, we performed gene set enrichment analysis (GSEA) to identify differences in molecular characteristics between these two clusters. After multiple testing corrections using the Benjamini-Hochberg FDR method, 822 and 23 categories were significantly overrepresented in cluster 1 when we used GO gene sets and HALLMARK gene sets in GSEA, respectively. On the other hand, no category was significantly overrepresented in cluster 2 (Supplementary Data 1). Significant enrichment of epidermis-associated pathways (GO0008544, GO0045682, GO0045684, and GO0043588) was observed in SCC- the top 1000 variable genes of all samples $(n=2322)$ is shown. The color and legend in each plot show the primary site and histology, respectively. d Ten top-ranked overrepresented pathways in cluster 1 based on GSEA by using HALLMARK gene sets and GO gene sets are shown.

dominant cluster 1 (Fig. 2d). Significant enrichments of p53 signaling, PI3K-AKT-mTOR signaling, and cell cycle signaling were also observed in SCC-dominant cluster 1, which corresponded to frequent genomic alterations in MCT-SCC.

\section{Similarity among pan-SCC based on integrated analyses of transcriptomic and immunohistochemistry data}

Although human papilloma virus (HPV) infection is associated with the occurrence of oropharyngeal cancer and cervical cancer, there were no obvious HPV-positive cases in any MCT-SCC/ASC samples, which is in line with a previous comprehensive study [5]. Then, we focused on HPV-negative SCC because having well known that HPVnegative tumors have different biological characteristics compared with HPV-positive cancers [17, 26].

We used the top 1000 variable genes in 1030 curated SCC samples to conduct hierarchical clustering and PCA plots. By hierarchical clustering analysis, SCC samples were divided into two clusters (cluster L and cluster R) mainly by primary tumor site (Fig. 3a, b). All MCT-SCCs were located in cluster $\mathrm{R}$, which mainly consisted of lung SCC. On the other hand, all cutaneous SCCs were classified 
a

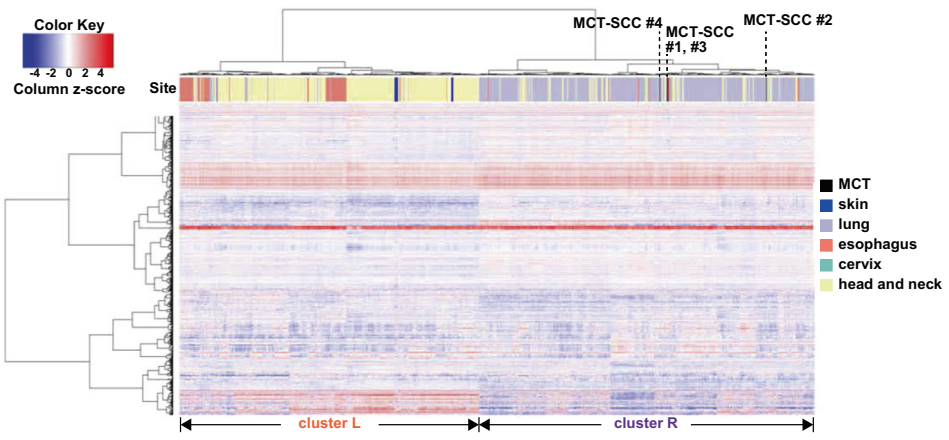

b

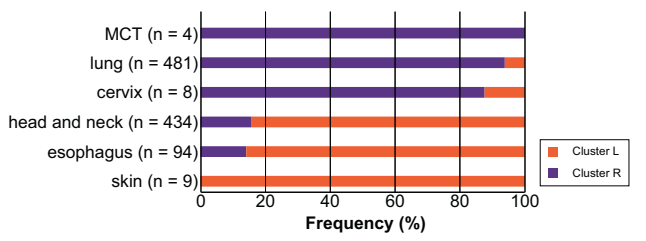

C

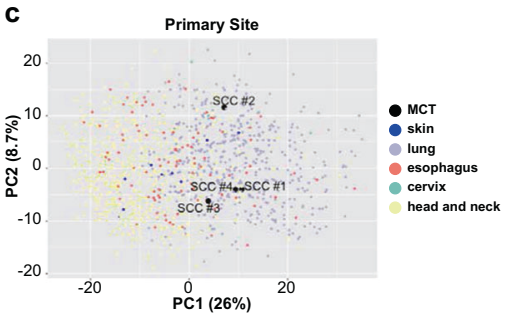

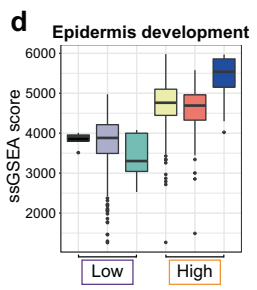
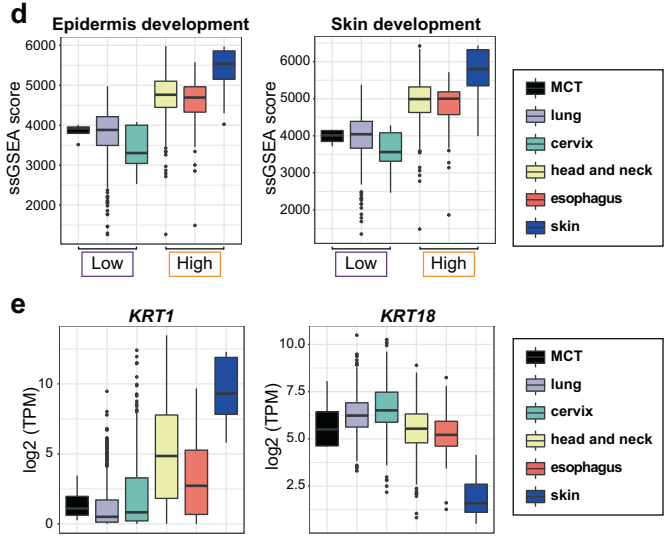

f

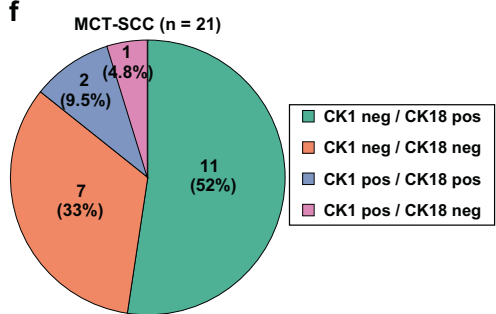

Fig. 3 Similarity among pan-SCCs based on integrated analyses of transcriptomic and immunohistochemistry data. a A heatmap demonstrates the hierarchical clustering using the top 1000 variable genes in 1030 SCC patients. The color bar and legend show the primary site of the tumor. $\mathbf{b}$ The distribution of clusters $\mathrm{L}$ and $\mathrm{R}$ per tumor type stratified by histological types is shown. c PCA using the variable 1000 genes of 1030 SCC patients is shown. The color and

in cluster L, which consisted of major parts of head and neck SCCs and esophagus SCCs. A PCA plot using the same 1000 variable genes also demonstrated the distinction between head and neck and lung SCC (Fig. 3c). MCT-SCCs were located in close proximity to lung SCCs in the PCA plot. When we performed GSEA to use GO gene sets and HALLMARK gene sets, 18 and 8 categories were significantly overrepresented in cluster L, respectively (Supplementary Data 2). No category was significantly overrepresented in cluster R. Interestingly, 15 of 18 (83\%) overrepresented GO categories in cluster L were common to overrepresented in cluster 1 (SCC-dominant cluster). Similarly, five of eight (63\%) overrepresented categories of HALLMARK gene sets in cluster L were in common with those of cluster 1 (SCC-dominant cluster) (Supplementary Fig. 4). In particular, epidermis-associated pathways were commonly overrepresented in both cluster $\mathrm{L}$ and cluster 1 . Then, we performed single sample GSEA (ssGSEA) to compare the pathway activity of two representative epidermis-associated pathways (GO:0008544 and GO:00043588) in different types of SCCs. Based on the above results, SCCs were divided into two epidermisassociated pathways activity subgroups: high (head and neck, esophagus, and skin) and low (cervix, lung, and MCT) (Fig. 3d, Supplementary Fig. 5). legend in each plot show the primary site of the tumor. d Box plots show ssGSEA score of epidermis development and skin development in different SCCs. e Box plots show log2 TPM of KRT1 and KRT18 in different SCCs. $\mathbf{f}$ Pie charts show the number and percentage of CK1/ CK18 protein expression evaluated by using immunohistochemistry in 21 MCT-SCCs.

For further characterization of MCT-SCC, we focused on cytokeratin genes composed of epidermis-associated pathways. The cytokeratin gene (KRT1-20) expression pattern was obviously different between SCC and AC (Supplementary Fig. 6). The expression patterns of KRT1 and KRT18 were clearly different between MCT-SCC and cutaneous SCC (Fig. 3e). Next, we performed immunohistochemistry on an additional 21 MCT-SCC FFPE samples by using CK1 and CK18 antibodies. CK1 and CK18 were expressed in 3/21 (14\%) and 13/21 (62\%) of MCT-SCCs, and the skin SCC pattern (CK1 positive and CK18 negative) was observed in only one MCT-SCC sample (Fig. 3f).

\section{Identification of XCL1 overexpressed specifically in MCT-SCC}

We analyzed differentially expressed genes (DEGs) between MCT-SCC and each of the other SCCs arising from different anatomical sites. Compared with all types of SCCs arising from various anatomical sites, 29 genes were differentially expressed in MCT-SCC (Fig. 4a). Of these, $X C L 1$ was stably overexpressed in MCT-SCC compared with other SCCs. XCL1 was overexpressed in MCT-SCC and MCT-ASC but not in MCT-AC (Fig. 4b). To validate the specificity of XCL1 overexpression in MCT-SCC/ASC, 
a

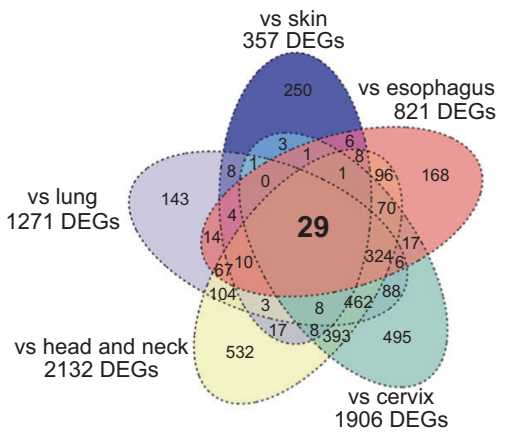

b

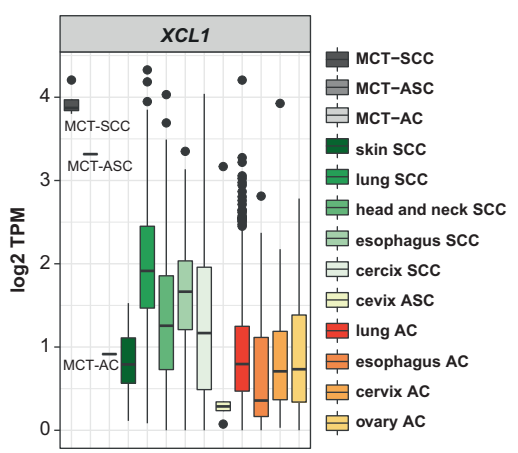

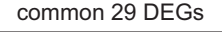

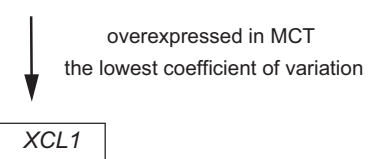

$X C L 1$ d

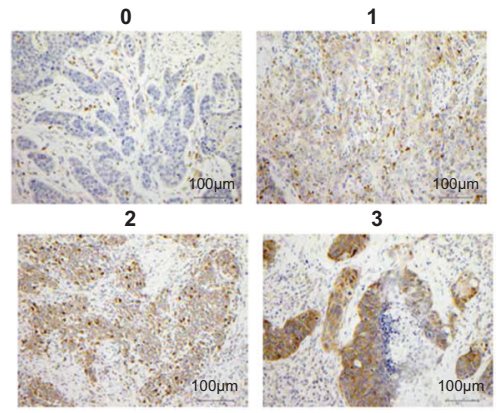

\section{c}

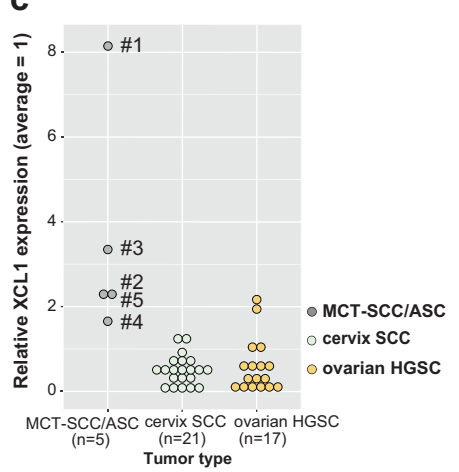

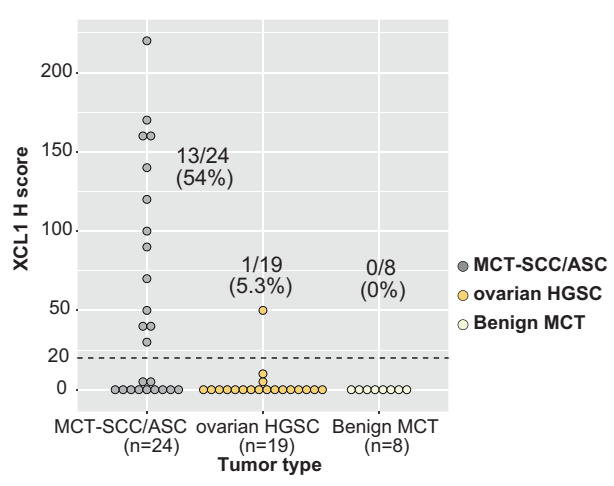

Fig. 4 Identification of XCLI overexpressed specifically in MCTSCC. a Venn diagram shows the number of differentially expressed genes (DEGs) between MCT-SCC and each SCC using TCC based on an FDR $q$ value $<0.05$. b Box plots show $\log 2$ TPM of XCL1 in different types of SCCs, ASCs, and ACs. c Dot plots show relative $X C L 1$ expression among MCT-SCC/ASC $(n=5)$, cervix SCC $(n=$ $21)$, and ovarian HGSC $(n=17)$ samples by using real-time RT-PCR.

we used 5 MCT-SCC/ASCs, 21 cervical SCCs, and 17 ovarian high-grade serous carcinoma (HGSC) samples to perform quantitative RT-PCR for XCL1 mRNA (Fig. 4c). MCC-SCC/ASC had higher expression of XCL1 compared with that of cervical SCC or ovarian HGSC. To confirm the protein-level expression and localization of XCL1 in tumor tissue, we added immunohistochemistry for 24 MCT-SCC/ ASC and 19 HGSC samples. XCL1 staining was observed in both MCT-SCC cells and some intratumor immune cells (Supplementary Fig. 7). XCL1 expression in tumor cells was detected in 13 out of 24 MCT-SCC/ASCs (54\%) and 1 out of 19 HGSCs (5.3\%). No obvious positive lesion was detected in eight benign MCTs (Fig. 4d and Supplementary Fig. 8).

\section{Clinical significance of XCL1 overexpression in MCT- SCC}

To assess the clinical significance of XCL1 in MCT-SCC, we divided 24 MCT-SCC/ASC samples into two groups based on XCL1 expression level. Although nonkeratinizing tumors were significantly more frequent in the XCL1-high group than in the XCL1-low group $(p=0.019)$, there was d Dot plots show XCL1 expression of MCT-SCC/ASC $(n=24)$, ovarian HGSC $(n=19)$, and benign MCT $(n=8)$ samples evaluated by using immunohistochemistry. The $\mathrm{H}$ score was obtained by the following formula: $3 \times$ percentage of strongly stained cytoplasm $+2 \times$ percentage of moderately stained cytoplasm + percentage of weakly stained cytoplasm, giving a range of $0-300$. The dotted line indicates the lower cutoff value for a positive result $(\mathrm{H}$ score $=20)$. no association between XCL1 expression and other clinicopathological findings in MCT-SCC/ASC (Supplementary Table 8). Moreover, there was no significant difference in progression-free or overall survival between the XCL1high and -low groups (Supplementary Fig. 9). The XCL1-XCR1 axis plays crucial roles in the development of efficient cytotoxic immunity [27]. Therefore, we investigated the associations of XCL1 expression on tumor cells with tumor infiltration of CD8-positive T cells and PD-L1 expression on tumor cells, which is known as a biomarker for immune checkpoint inhibitors [28]. More than half of the MCT-SCC/ASCs [14/24 (58\%)] showed a high number of tumor-infiltrating CD8-positive T cells (Fig. 5a), and the CD8-high group showed a significantly better prognosis than the CD8-low group (Fig. 5b). On the other hand, PD$\mathrm{L} 1$ expression on more than $1 \%$ of tumor cells was observed in 15 out of 24 MCT-SCC/ASCs (63\%). Of them, six samples showed PD-L1 expression on over $50 \%$ of tumor cells (Fig. 5c). There was no significant association between PD-L1 expression on tumor cells and the prognosis in MCT-SCC/ASC (Fig. 5d). We performed multivariate analysis by using XCL1, CD8, and PD-L1 expression. Of three factors, only CD8 expression was significantly 
a

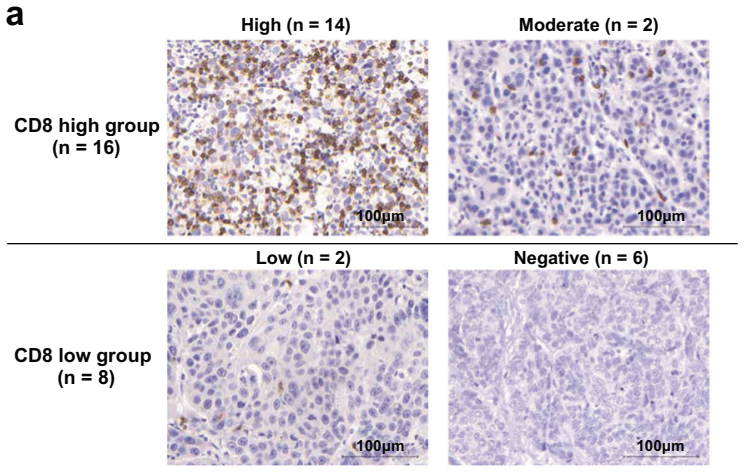

C
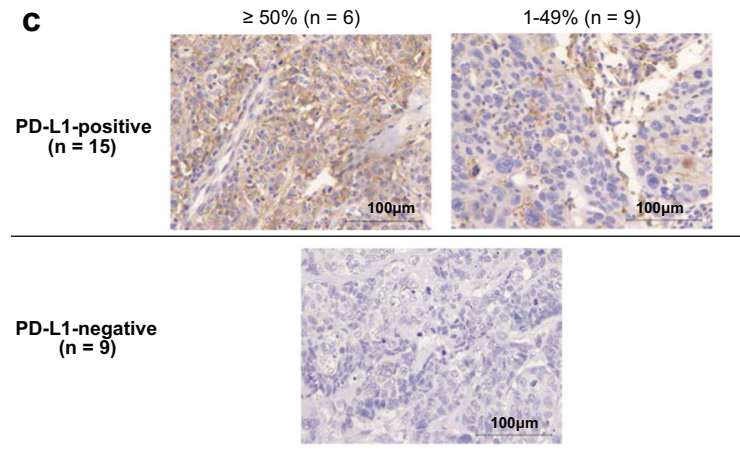

Fig. 5 The clinical significance of the number of tumor-infiltrating CD8-positive lymphocytes and PD-L1 expression on tumor cells in 24 MCT-SCC/ASCs. a Immunohistochemical staining for CD8 in 24 MCT-SCC/ASCs. b Kaplan-Meier estimates of progression-free

associated with both progression-free survival and overall survival (Supplementary Table 9). Intriguingly, 11 of 13 (85\%) XCL1-positive MCT-SCC/ASCs showed a high tumor infiltration rate of CD8-positive T cells and PD-L1 expression on tumor cells (Fig. 6a). A significant association between XCL1 expression and a high tumor infiltration rate of CD8-positive T cells was observed in MCT-SCC/ ASCs $(p=0.0078)$ (Fig. 6b). In addition, there was a significant association between XCL1 expression and PD-L1 expression in MCT-SCCs $(p=0.0022)$. Subsequently, we evaluated relationships between gene expression of XCL1 and $C D 8 A$ or $C D 274$ which encodes PD-L1 in pan-SCCs and other types of SCCs by using publicly available RNAsequencing data. No significant correlations between XCL1 and $C D 8 A / C D 274$ in pan-SCCs nor any other types of SCCs were observed (Supplementary Fig. 10).

\section{Discussion}

In this study, we demonstrated unique gene expression profiles in carcinomas arising from MCT. In particular, XCL1 was specifically overexpressed in MCT-SCC/ASC but not in SCC and AC derived from other anatomical sites and benign MCT. XCL1 expression on tumor cells was significantly associated with the number of tumor- b
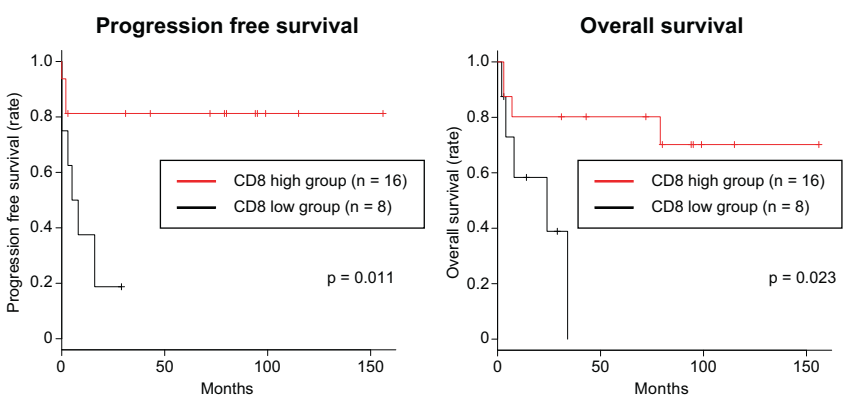

d
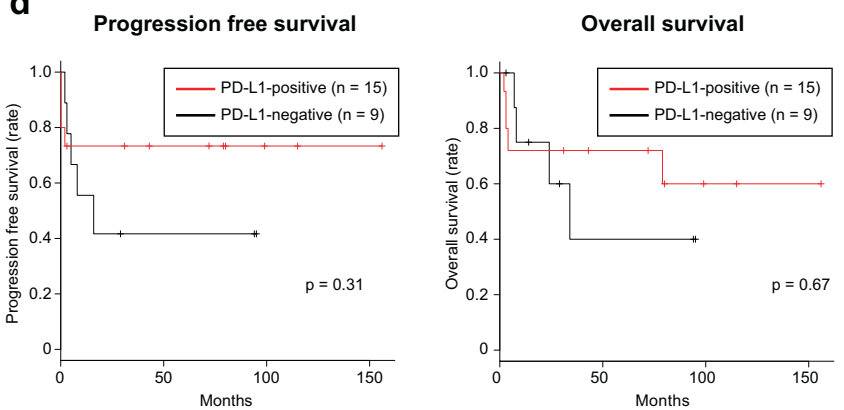

survival and overall survival between the CD8-high and -low groups. c Immunohistochemical staining for PD-L1 in 24 MCT-SCC/ASCs. d Kaplan-Meier estimates of progression-free survival and overall survival between PD-L1-positive and PD-L1-negative patients.

infiltrating CD8-positive T cells and PD-L1 expression on tumor cells. Our findings suggest that XCL1 expression may be a novel biomarker for the diagnosis of malignant transformation of MCT into SCC and a biomarker candidate for therapeutic response to an anti-PD-1/PD-L1 therapy.

Consistent with a previous report [5], we detected a high frequency of TP53 and PIK3CA (E542K/E545K) mutations in MCT-SCCs. Our exome sequencing analysis showed that no other genes were mutated in more than $50 \%$ of MCTSCC. TP53 and PIK3CA mutations might have a crucial role in SCC transformation from MCT [5]. In particular, most MCT-SCCs [7/8 (87.5\%)] harbored at least one known oncogenic alteration in the PI3K-AKT-mTOR pathway that was targeted by many inhibitors [29]. In addition, we identified oncogenic kinase gene alterations that were druggable, and the clinical efficacy of the molecular target therapy was confirmed in various types of malignancies [19], such as FGFR3-TACC3 fusion, BRAF missense mutation, FLT3 missense mutation, and MET amplification. Intriguingly, the SETD2 nonsense mutation was recurrently detected in MCT-SCC, including sample $\# 1$, in which no druggable alteration was identified. SETD2 is a histone-modifying enzyme responsible for all trimethylation of H3K36 (H3K36me3). Decreases in H3K36me3 lead to chromosomal instability, such as MMRdeficient tumors [30], and cause drug vulnerabilities [31]. 


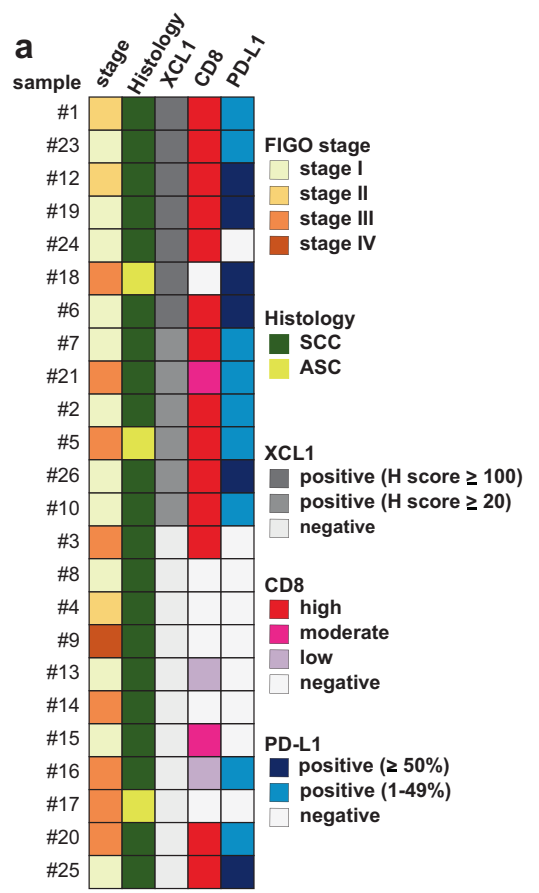

b
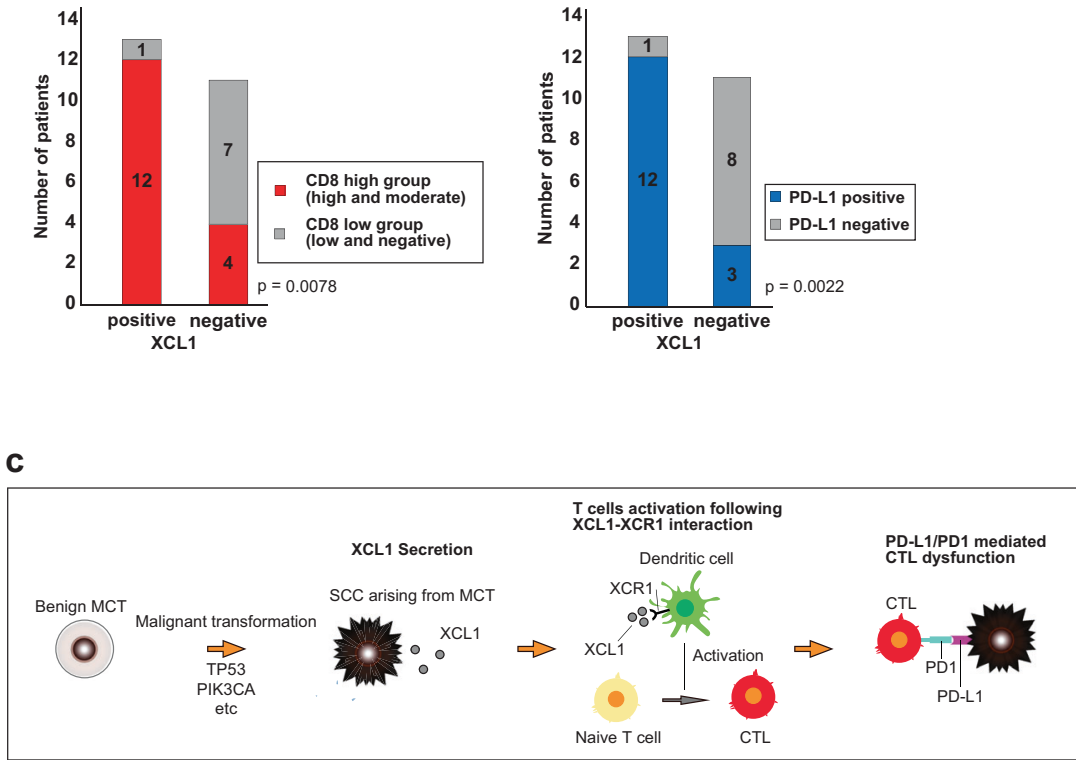

Fig. 6 Clinical significance of XCL1 overexpression in MCT-SCC. a Heatmaps demonstrate both clinical information and immunohistochemical findings in 24 MCT-SCC/ASCs. b Bar graphs show tumorinfiltrating CD8-positive T cells and PD-L1 expression in MCT-SCC/

Therefore, some MCT-SCC patients might obtain a new therapeutic strategy for SETD2 mutation in the future. We evaluated TMB in eight MCT-SCC samples, and there was no obvious hypermutated tumor in our samples compared with other types of cancer [24]. Our findings suggest that integrated genomic and transcriptomic analysis could increase therapeutic drug targets in MCT-SCC.

We identified unique gene expression profiles in carcinomas arising from MCT by comparing with pan-cancer RNAsequencing data. Three histological types of carcinomas (SCC, ASC, and AC) arising from MCT showed different gene expression patterns. Our data suggested that the expression profile of MCT-SCC was similar to that of SCCs arising from other anatomical sites. In particular, MCT-SCC showed a more similar expression pattern to lung SCC than cutaneous SCC. The origin of MCT-SCC has been discussed for decades, and there are two possible origins: epidermis [4, 32] and columnar epithelium [33, 34]. The epidermis origin theory is the most likely candidate because skin epithelium is generally found in MCT. Moreover, carcinoma in situ with a Bowen's disease-like pattern that is typical in cutaneous SCC is found in some cases of MCT-SCCs [4, 32]. On the other hand, the columnar epithelium origin theory has also been considered because some MCT-SCCs exhibited a sequential change from respiratory epithelium to carcinoma in situ [33]. CK18-positive and CK10-negative expression patterns that were observed in lung and cervix SCCs were found in the majority of MCT-SCCs [34]. Although we could
ASCs between XCL1-positive and XCL1-neagtive patients. c Our hypothesis of PD-L1/PD1-mediated cytotoxic T-lymphocyte dysfunction following XCL1 production by MCT-SCC is shown.

not find a sequential change from columnar epithelium to carcinoma in situ in our H\&E slides of MCT-SCC samples, our results of expression data analysis support the columnar epithelium origin theory in MCT-SCC.

Although the gene expression profile of MCT-SCC was similar to that of lung SCC, we identified that XCL1 was specifically overexpressed in MCT-SCC compared with other SCCs and benign MCT. There were no significant differences in prognosis or clinical stage between the high- and lowXCL1 groups in MCT-SCCs. These results suggest that XCL1 may be a biomarker for SCC transformation from MCT but not a prognostic factor. XCL1 is a C class chemokine and is produced mainly by natural killer and activated CD8-positive T cells $[35,36]$. The selective receptor XCR1 is expressed by a subpopulation of dendritic cells, and the XCL1-XCR1 axis plays a crucial role in the development of efficient cytotoxic immunity [27]. In addition, the XCL1-XCR1 interaction is involved in tumor migration, invasion, and proliferation in several malignancies [37, 38]. In MCT-SCC, we found XCL1 expression on tumor cells as well as some intratumor immune cells in XCL1-positive MCT-SCCs/ASCs. These intratumor immune cells with XCL1 expression were rarely observed in HGSCs. These findings might reflect that $\mathrm{CD} 8$-positive $\mathrm{T}$ cells activated by the XCL1-XCR1 interaction produced XCL1 [27].

Immunohistochemistry analysis demonstrated that XCL1 expression on tumor cells was positively correlated with the number of tumor-infiltrating CD8-positive $\mathrm{T}$ cells and 
PD-L1 expression on tumor cells, which were predictive biomarker candidates in an immune checkpoint therapy in various types of malignancies. Based on tumor PD-L1 expression and the presence of tumor-infiltrating lymphocytes, four different types of tumor immune microenvironment were proposed to predict the response to immune checkpoint therapies [28]. More than half of MCT-SCCs [13/24, (54\%)] showed high tumor infiltration of CD8positive T cells and high PD-L1 expression, and these cases might be treated effectively by the PD1/PD-L1 blockage therapy. XCL1 expression may be a biomarker candidate to select an appropriate therapy for MCT-SCC patients.

On the other hand, 11/24 (46\%) MCT-SCC patients with low or negative tumor-infiltrating CD8-positive T cells or low PD-L1 expression tended to have poor prognosis. For these patients, other strategies such as combination therapy that is designed to bring $\mathrm{T}$ cells into tumors and avoid them being turned off, such as the combination of anti-CTLA-4 and anti-PD-1, might be needed [28].

No significant correlations between $X C L 1$ expression and $\mathrm{CD} 8 \mathrm{~A} / \mathrm{CD} 274$ expression in pan-SCC data nor any types of SCCs were observed. Although we could not investigate the association of XCL1 expression with CD8-positive T cells infiltration/PD-L1 expression in the protein level in other SCCs, these associations might be specific to MCT-SCC. Moreover, the number of XCL1-high samples comparable to MCT-SCC in other SCCs was quite limited. Further analysis should be performed to reveal whether the association is also observed in other types of SCCs.

Our hypothesis of the tumor immune microenvironment in MCT-SCC is summarized in Fig. 6c. However, there are several important limitations in this study. Because of the tumor rarity, our sample size was small. In addition, we were not able to obtain any resources of MCT-SCC, such as the cancer cell line, for the purpose of performing functional analysis, especially the XCL1-XCR1 interaction in MCTSCC. Further studies, including large-scale genomic and transcriptomic analyses and in vitro/vivo experiments, are required to prove our hypothesis.

In conclusion, our comprehensive genomic and transcriptomic analysis clarified the molecular characteristics of carcinomas arising from MCT. XCL1 expression might be a promising biomarker for malignant transformation of $\mathrm{MCT}$ into SCC and a biomarker candidate of therapeutic response to an anti-PD1/PD-L1 therapy.

\section{Materials and methods}

\section{Clinical samples}

This study was performed in accordance with the Declaration of Helsinki and was approved by the institutional ethics review board at Niigata University, Osaka University, Jichi Medical University, Niigata Cancer Center, and National Institute of Genetics. A total of 27 patients histologically diagnosed with carcinoma arising from MCT of the ovary between 1999 and 2018 at the Niigata University, Osaka University, Jichi Medical University, and Niigata Cancer Center were enrolled. Fresh frozen tumor tissues and FFPE samples obtained by surgical resection were collected. In addition, fresh frozen tumor tissues and FFPE samples from patients who were diagnosed with high-grade serous ovarian cancer, cervical cancer, and MCT of the ovary at Niigata University in the same period were also collected as controls. All patients provided informed consent for the collection of samples and subsequent analysis.

\section{Whole-exome sequencing and analysis}

Before sequencing analysis, we confirmed that all fresh frozen samples histologically contained over $50 \%$ of tumor cells (Supplementary Fig. 11). Two fresh frozen samples (samples \#15 and \#21) were excluded because of low tumor cell content. We extracted DNA from tumor tissues and blood samples as previously described [39]. We performed whole-exome sequencing using genomic DNA derived from the five carcinomas arising from MCT samples [one sample with matched blood sample (sample \#1) and four samples (sample \#2, \#3, \#4, and \#5) without matched blood samples], and detected putative somatic mutations and copy number alterations. The details of experimental methods for whole-exome sequencing and computational analyses have been previously described $[39,40]$.

\section{Detection of putative somatic mutations and copy number alterations}

Detection of putative somatic mutations and copy number alterations in sample \#1 was conducted as previously described [39]. For samples without matched blood samples (samples \#2, \#3, \#4, and \#5), the detection of putative somatic mutations and copy number alterations was also performed as previously described [40]. We focused on mutations and copy number alterations annotated in OncoKB [19] as pathogenic (oncogenic, likely oncogenic, and predicted oncogenic) at August 6, 2019. We also focused on mutations that were annotated in COSMIC [20], release v87 as pathogenic (FATHHMM score $\geq 0.7$ ). We defined a threshold of $\geq 6$ copies as gene amplification. Homozygous deletion was defined as a copy number equal to zero. To validate homozygous deletions, we examined the mRNA expression of homozygously deleted genes by using RNA-sequencing data. We excluded homozygously deleted genes showing obvious mRNA expression despite copy number 0 from copy number alteration analysis. 


\section{Tumor mutation burden (TMB)}

The TMB, defined as the rate of nonsynonymous single nucleotide variants (SNVs) per megabase, was determined for samples analyzed by using whole-exome sequencing. To estimate the TMB, only SNVs that covered at least ten reads in coding area were evaluated.

\section{Target sequencing analysis (CANCERPLEX ${ }^{\circledR}$ )}

Six FFPE MCT-SCC samples (samples \#6, \#7, \#8, \#23, $\# 24$, and \#25) obtained by surgical resection at the Niigata Cancer Center were analyzed by a gene panel containing

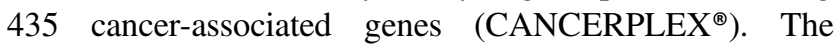
details of the 435 genes are shown in https://kewinc.com/w p-content/uploads/2017/07/Full-gene-list.pdf. An independent pathologist evaluated tumor content, and over $20 \%$ of tumor content was confirmed in all samples. DNA was extracted using the BioStic FFPE Tissue DNA Isolation Kit (MO BIO Laboratories, Carlsbad, CA, USA). Three FFPE samples (samples \#23, \#24, and \#25) were excluded because of poor DNA quality. All sample preparation and genomic sequencing and analysis were performed in a CLIA/CAP-accredited laboratory (KEW, Cambridge, MA, USA). The details of experimental methods for nextgeneration sequencing and computational analyses are the same as previously described [41].

\section{Pan-cancer data analysis}

Mutation data of TCGA samples were downloaded from TCGA Pan Cancer Atlas Studies. Mutation data of cutaneous SCC were downloaded from MSK-IMPACT using cBioPortal (http://www.cbioportal.org) [42]. The HPV status of TCGA SCC samples was downloaded from a previous study [11].

\section{RNA sequencing}

Total RNA was extracted from frozen samples using TRIzol (Invitrogen, Carlsbad, CA, USA).

Total RNA was used for the library preparation, which was conducted using a TruSeq Stranded mRNA Library Prep Kit (Illumina, San Diego, CA, USA) according to the manufacturer's protocol. The samples were sequenced on the Illumina HiSeq 2500 platform with the $2 \times 100$-bp paired-end read module. The details of the procedure were the same as previously described [40].

\section{Detection of fusion transcripts}

To identify fusion transcripts, we used both PRADA [21, 40] and FusionCatcher [22] as previously described.

\section{RNA-sequencing data analysis}

We downloaded RNA-sequencing data of cutaneous SCC (SRP078314) from Gene Expression Omnibus [43] and used kallisto (version 0.43.0) to perform TPM normalization of our RNA sequencing data from carcinoma arising from MCT samples as well as cutaneous SCC RNA sequencing data [44]. Gene expression data of TCGA pancancer samples calculated by kallisto (version 0.43.0) were downloaded from the Google Cloud Pilot RNA sequencing for CCLE and TCGA (Tatlow, PJ. 2016. "Google Cloud Pilot RNA-Sequencing for CCLE and TCGA". OSF. July 19. osf.io/gqrz9.). To obtain one expression value per gene and sample, transcripts measuring the same gene were averaged. Then, we calculated the median absolute deviation to exclude variable genes across all samples. We performed clustering analysis of both PCA and generated a heatmap using the $\log 10$ transformed TPM value of the top 1000 variable genes across all samples. The hierarchical clustering analysis was performed by applying the absolute Pearson distance measure and Ward D algorithm. The calculated distance was plotted using the heatmap.3R package (https://www.rdocumentation.org/packages/GMD/versions/ 0.3.3/topics/heatmap.3). PCA was performed using the "prcomp" package in $\mathrm{R}$ version 3.5.2. We evaluated the validity of cluster number using both the Calinski and Harabasz index [45] and the Kzanwski and Lai index [46] (Supplementary Fig. 12). We performed GSEA using HALLMARK gene sets and GO gene sets that were downloaded from the Molecular Signatures Database (http://www.broad.mit.edu/gsea/.msigdb/msigdb_index.

html). By using a normalized enrichment score and $q$ value, we extracted overrepresented pathways for which the $-\log 2$ ( $q$ value) was $>2.0$. We ran ssGSEA using GO gene sets for each sample. We used the TCC R package [47] to detect DEGs $(q$ value $<0.05)$ between MCT-SCC and each group of SCC arising from other anatomical sites. To identify important DEGs, we calculated the coefficient of variation (the ratio of the standard deviation to the mean).

\section{Quantitative real-time RT-PCR}

We performed quantitative real-time RT-PCR as previously reported [40]. All primers used are shown in Supplementary Table 10.

\section{HPV genotyping}

For five frozen samples (samples \#1, \#2, \#3, \#4, and \#5), HPV-DNA testing targeting 16 high- and low-risk HPV genotypes (genotypes $6,11,16,18,30,31,33,35,39,45$, $51,52,56,58,59$, and 66) was performed using the multiplex PCR method (PapiPlex) at the GLab Pathology 
Center Co., Ltd (Sapporo, Japan) [48]. In addition, for three FFPE samples (sample \#6, \#7, and \#8), HPV-16 and HPV18 viral sequences were analyzed by using CANCERPLEX $^{\circledast}$ as previously described [23].

\section{Histological analysis}

All histological specimens were reviewed by two gynecological pathologists (TM and TE). In immunohistochemical analysis, we excluded two stage IA MCT-SCC samples (sample \#11 and sample \#22) that did not contain enough tumor cells. Antibodies were purchased from the indicated suppliers: CD8 (M7103, Dako; dilution ratio 1:100), CK1 (sc-376224, Santa Cruz; dilution ratio 1:50), CK18 (ab668, Abcam; dilution ratio 1:100), PD-L1 (ab205921, Abcam; dilution ratio 1:500), and XCL1 (HPA057725, Atlas Antibodies; dilution ratio 1:200). We experimented with immunohistochemical staining as previously reported [40]. CK1 and CK18 were defined as "positive" when $>10 \%$ of the tumor cells showed cytoplasmic immunoreactivity [34]. Tumor-infiltrating CD8-positive $\mathrm{T}$ cells were manually counted in three random high-power fields. Tumorinfiltrating $\mathrm{T}$ cells were graded as negative, low, moderate, or high $(0, \leq 5,6-19$, or $\geq 20 \mathrm{~T}$ cells per high-power field, respectively) [49]. Over 1\% of PD-L1 expression on tumor cells was defined as "positive" based on the previous assessment [50]. Cytoplastic staining of XCL1 on tumor cells was evaluated on a scale of 0 (no staining) to 3 (strong staining). Subsequently, the $\mathrm{H}$ score was obtained by the formula: $3 \times$ percentage of strongly stained cytoplasm + $2 \times$ percentage of moderately stained cytoplasm + percentage of weakly stained cytoplasm, giving a range of $0-300$ [51]. When the $\mathrm{H}$ score was higher than 20 , XCL1 was defined as "positive".

\section{Statistical analysis}

All computations were conducted using $\mathrm{R}$ ( $\mathrm{R}$ Core Team (2018): a language and environment for statistical computing. R Foundation for Statistical Computing, Vienna, Austria, http://www.R-project.org/). Fisher's exact test was used to evaluate the significance between data groups. Spearman's rank test was used to evaluate correlation between data groups. $P$ values $<0.05$ indicated statistical significance. Standard statistical tests were used as appropriate, including unpaired $t$-test and $\log$ rank test. Cox proportional model was used for the multivariate analysis.

Acknowledgements We are grateful to Anna Ishida, Junko Kajiwara, Junko Kitayama, and Yumiko Sato for their technical assistance. We also grateful to Yusuke Tanaka, Reisa Kakubari, Mariko Kayamori, and Taeko Hyuga for sample collections. This work was supported in part by JSPS KAKENHI grant number 18K16760 (Grant-in-Aid for
Young Scientists for RT), JP16H06267 (Grant-in-Aid for Young Scientists A for KY), JP16H06279 (Grant-in-Aid for Scientific Research on Innovative Areas-Platforms for Advanced Technologies and Research Resources for HN and KY), the Mochida Grant for KY, the Tsukada Grant for Niigata University Medical Research for RT, and "Challenging Exploratory Research Projects for the Future" grant from ROIS (Research Organization of Information and Systems) for $\mathrm{HN}$.

Author contributions RT, KY, and TE designed the research plan. RT, KY, NY, MY, HI, KH, AK, YU, YT, HF, and TW collected tissue samples. HN and II performed sequencing experiments. RT, KY, HN, and SO conducted sequencing data analysis. RT, KN, and TM reviewed pathological findings. RT and NY conducted the experiments. RT and KY wrote the main manuscript and prepared all figures and tables. RT, KY, HN, and TE discussed the results. All authors reviewed and approved the final manuscript.

\section{Compliance with ethical standards}

Conflict of interest The authors declare that they have no conflict of interest.

Publisher's note Springer Nature remains neutral with regard to jurisdictional claims in published maps and institutional affiliations.

Open Access This article is licensed under a Creative Commons Attribution 4.0 International License, which permits use, sharing, adaptation, distribution and reproduction in any medium or format, as long as you give appropriate credit to the original author(s) and the source, provide a link to the Creative Commons license, and indicate if changes were made. The images or other third party material in this article are included in the article's Creative Commons license, unless indicated otherwise in a credit line to the material. If material is not included in the article's Creative Commons license and your intended use is not permitted by statutory regulation or exceeds the permitted use, you will need to obtain permission directly from the copyright holder. To view a copy of this license, visit http://creativecommons. org/licenses/by/4.0/.

\section{References}

1. Hackethal A, Brueggmann D, Bohlmann MK, Franke FE, Tinneberg HR, Munstedt K. Squamous-cell carcinoma in mature cystic teratoma of the ovary: systematic review and analysis of published data. Lancet Oncol. 2008;9:1173-80.

2. Mori Y, Nishii H, Takabe K, Shinozaki H, Matsumoto N, Suzuki K, et al. Preoperative diagnosis of malignant transformation arising from mature cystic teratoma of the ovary. Gynecol Oncol. 2003;90: $338-41$.

3. Akazawa M, Onjo S. Malignant transformation of mature cystic teratoma: is squamous cell carcinoma different from the other types of neoplasm? Int J Gynecol Cancer. 2018;28:1650-6.

4. Amerigo J, Nogales FF Jr, Fernandez-Sanz J, Oliva H, Velasco A. Squamous cell neoplasms arising from ovarian benign cystic teratoma. Gynecol Oncol. 1979;8:277-83.

5. Cooke SL, Ennis D, Evers L, Dowson S, Chan MY, Paul J, et al. The driver mutational landscape of ovarian squamous cell carcinomas arising in mature cystic teratoma. Clin Cancer Res. 2017;23:7633-40.

6. Cancer Genome Atlas Research Network. Comprehensive genomic characterization of squamous cell lung cancers. Nature. 2012;489:519-25. 
7. Cancer Genome Atlas Network. Comprehensive genomic characterization of head and neck squamous cell carcinomas. Nature. 2015;517:576-82.

8. Cancer Genome Atlas Research Network. Integrated genomic and molecular characterization of cervical cancer. Nature. 2017;543:378-84.

9. Cancer Genome Atlas Research Network. Integrated genomic characterization of oesophageal carcinoma. Nature. 2017;541: $169-75$.

10. Hoadley KA, Yau C, Hinoue T, Wolf DM, Lazar AJ, Drill E, et al. Cell-of-origin patterns dominate the molecular classification of 10,000 tumors from 33 types of cancer. Cell. 2018;173:291. e6-304.e6.

11. Campbell JD, Yau C, Bowlby R, Liu Y, Brennan K, Fan H, et al. Genomic, pathway network, and immunologic features distinguishing squamous carcinomas. Cell Rep. 2018;23:194.e6-212.e6.

12. Reck M, Rodriguez-Abreu D, Robinson AG, Hui R, Csoszi T, Fulop A, et al. Pembrolizumab versus Chemotherapy for PD-L1positive non-small-cell lung cancer. N Engl J Med. 2016;375: 1823-33.

13. Ferris RL, Blumenschein G, Jr., Fayette J, Guigay J, Colevas AD, Licitra L, et al. Nivolumab for recurrent squamous-cell carcinoma of the head and neck. N Engl J Med. 2016;375:1856-67.

14. Chung HC, Ros W, Delord JP, Perets R, Italiano A, ShapiraFrommer R, et al. Efficacy and safety of pembrolizumab in previously treated advanced cervical cancer: results from the Phase II KEYNOTE-158 Study. J Clin Oncol. 2019;37:1470-8.

15. Kudo T, Hamamoto Y, Kato K, Ura T, Kojima T, Tsushima T, et al. Nivolumab treatment for oesophageal squamous-cell carcinoma: an open-label, multicentre, phase 2 trial. Lancet Oncol. 2017;18:631-9.

16. Migden MR, Rischin D, Schmults CD, Guminski A, Hauschild A, Lewis $\mathrm{KD}$, et al. PD-1 blockade with cemiplimab in advanced cutaneous squamous-cell carcinoma. N. Engl J Med. 2018;379: 341-51.

17. Li B, Cui Y, Nambiar DK, Sunwoo JB, Li R. The immune subtypes and landscape of squamous cell carcinoma. Clin Cancer Res. 2019;25:3528-37.

18. Chiang AJ, Chen MY, Weng CS, Lin $\mathrm{H}$, Lu CH, Wang PH, et al. Malignant transformation of ovarian mature cystic teratoma into squamous cell carcinoma: a Taiwanese Gynecologic Oncology Group (TGOG) study. J Gynecol Oncol. 2017;28:e69.

19. Chakravarty D, Gao J, Phillips SM, Kundra R, Zhang H, Wang J, et al. OncoKB: a precision oncology knowledge base. JCO Precis Oncol. 2017;2017. https://doi.org/10.1200/PO.17.00011.

20. Forbes SA, Beare D, Gunasekaran P, Leung K, Bindal N, Boutselakis $\mathrm{H}$, et al. COSMIC: exploring the world's knowledge of somatic mutations in human cancer. Nucleic Acids Res. 2015;43:D805-11.

21. Torres-Garcia W, Zheng S, Sivachenko A, Vegesna R, Wang Q, Yao R, et al. PRADA: pipeline for RNA sequencing data analysis. Bioinformatics. 2014;30:2224-6.

22. Nicorici D, Şatalan M, Edgren H, Kangaspeska S, Murumägi A, Kallioniemi O, et al. FusionCatcher-a tool for finding somatic fusion genes in paired-end RNA-sequencing data. 2014. https:// doi.org/10.1101/011650.

23. Eifert C, Pantazi A, Sun R, Xu J, Cingolani P, Heyer J, et al. Clinical application of a cancer genomic profiling assay to guide precision medicine decisions. Per Med. 2017;14:309-25.

24. Yarchoan M, Hopkins A, Jaffee EM. Tumor mutational burden and response rate to $\mathrm{PD}-1$ inhibition. $\mathrm{N}$ Engl $\mathrm{J}$ Med. 2017;377:2500-1.

25. Cristescu R, Mogg R, Ayers M, Albright A, Murphy E, Yearley J, et al. Pan-tumor genomic biomarkers for PD-1 checkpoint blockadebased immunotherapy. Science. 2018;362:eaar3593.

26. Keck MK, Zuo Z, Khattri A, Stricker TP, Brown CD, Imanguli M, et al. Integrative analysis of head and neck cancer identifies two biologically distinct HPV and three non-HPV subtypes. Clin Cancer Res. 2015;21:870-81.

27. Dorner BG, Dorner MB, Zhou X, Opitz C, Mora A, Guttler S, et al. Selective expression of the chemokine receptor XCR1 on cross-presenting dendritic cells determines cooperation with CD8+ T cells. Immunity. 2009;31:823-33.

28. Teng MW, Ngiow SF, Ribas A, Smyth MJ. Classifying cancers based on T-cell infiltration and PD-L1. Cancer Res. 2015;75: 2139-45.

29. Fruman DA, Chiu H, Hopkins BD, Bagrodia S, Cantley LC, Abraham RT. The PI3K pathway in human disease. Cell. 2017; 170:605-35.

30. Li F, Mao G, Tong D, Huang J, Gu L, Yang W, et al. The histone mark H3K36me3 regulates human DNA mismatch repair through its interaction with MutSalpha. Cell. 2013;153:590-600.

31. Ding H, Zhao J, Zhang Y, Yu J, Liu M, Li X, et al. Systematic analysis of drug vulnerabilities conferred by tumor suppressor loss. Cell Rep. 2019;27:3331.e6-44.e6.

32. Marcial-Rojas RA, Medina R. Cystic teratomas of the ovary; a clinical and pathological analysis of two hundred sixty-eight tumors. AMA Arch Pathol. 1958;66:577-89.

33. Hirakawa T, Tsuneyoshi M, Enjoji M. Squamous cell carcinoma arising in mature cystic teratoma of the ovary. Clinicopathologic and topographic analysis. Am J Surg Pathol. 1989;13:397-405.

34. Iwasa A, Oda Y, Kaneki E, Ohishi Y, Kurihara S, Yamada T, et al. Squamous cell carcinoma arising in mature cystic teratoma of the ovary: an immunohistochemical analysis of its tumorigenesis. Histopathology. 2007;51:98-104.

35. Lei Y, Takahama Y. XCL1 and XCR1 in the immune system. Microbes Infect. 2012;14:262-7.

36. Kelner GS, Kennedy J, Bacon KB, Kleyensteuber S, Largaespada DA, Jenkins NA, et al. Lymphotactin: a cytokine that represents a new class of chemokine. Science. 1994;266:1395-9.

37. Khurram SA, Whawell SA, Bingle L, Murdoch C, McCabe BM, Farthing PM. Functional expression of the chemokine receptor XCR1 on oral epithelial cells. J Pathol. 2010;221:153-63.

38. Kim M, Rooper L, Xie J, Rayahin J, Burdette JE, Kajdacsy-Balla AA, et al. The lymphotactin receptor is expressed in epithelial ovarian carcinoma and contributes to cell migration and proliferation. Mol Cancer Res. 2012;10:1419-29.

39. Suda K, Nakaoka H, Yoshihara K, Ishiguro T, Tamura R, Mori Y, et al. Clonal expansion and diversification of cancer-associated mutations in endometriosis and normal endometrium. Cell Rep. 2018;24:1777-89.

40. Tamura R, Yoshihara K, Saito T, Ishimura R, Martinez-Ledesma JE, Xin $\mathrm{H}$, et al. Novel therapeutic strategy for cervical cancer harboring FGFR3-TACC3 fusions. Oncogenesis. 2018;7:4.

41. Ichikawa $H$, Nagahashi M, Shimada $Y$, Hanyu $T$, Ishikawa $T$, Kameyama H, et al. Actionable gene-based classification toward precision medicine in gastric cancer. Genome Med. 2017;9:93.

42. Cerami E, Gao J, Dogrusoz U, Gross BE, Sumer SO, Aksoy BA, et al. The cBio cancer genomics portal: an open platform for exploring multidimensional cancer genomics data. Cancer Disco. 2012;2:401-4.

43. Chitsazzadeh V, Coarfa C, Drummond JA, Nguyen T, Joseph A, Chilukuri S, et al. Cross-species identification of genomic drivers of squamous cell carcinoma development across preneoplastic intermediates. Nat Commun. 2016;7:12601.

44. Bray NL, Pimentel H, Melsted P, Pachter L. Near-optimal probabilistic RNA-seq quantification. Nat Biotechnol. 2016;34:525-7.

45. Caliński T, Harabasz J. A dendrite method for cluster analysis. Commun Stat Theory Methods. 1974;3:1-27.

46. Krzanowski WJ, Lai YT. A criterion for determining the number of groups in a data set using sum-of-squares clustering. Biometrics. 1988;44:23-4. 
47. Sun J, Nishiyama T, Shimizu K, Kadota K. TCC: an R package for comparing tag count data with robust normalization strategies. BMC Bioinform. 2013;14:219.

48. Nishiwaki M, Yamamoto $\mathrm{T}$, Tone $\mathrm{S}$, Murai $\mathrm{T}$, Ohkawara $\mathrm{T}$, Matsunami T, et al. Genotyping of human papillomaviruses by a novel one-step typing method with multiplex PCR and clinical applications. J Clin Microbiol. 2008;46:1161-8.

49. Zhang L, Conejo-Garcia JR, Katsaros D, Gimotty PA, Massobrio $\mathrm{M}$, Regnani $\mathrm{G}$, et al. Intratumoral T cells, recurrence, and survival in epithelial ovarian cancer. N Engl J Med. 2003;348:203-13.
50. O'Malley DP, Yang Y, Boisot S, Sudarsanam S, Wang JF, Chizhevsky V, et al. Immunohistochemical detection of PD-L1 among diverse human neoplasms in a reference laboratory: observations based upon 62,896 cases. Mod Pathol. 2019;32: 929-42.

51. John T, Liu G, Tsao MS. Overview of molecular testing in nonsmall-cell lung cancer: mutational analysis, gene copy number, protein expression and other biomarkers of EGFR for the prediction of response to tyrosine kinase inhibitors. Oncogene. 2009;28(Suppl 1):S14-23. 